\title{
Improving Industrial Chemical Processes via Novel Electron Beam Accelerator
}

\section{E-beam accelerators}

\section{an established industry tool}

Electron beams are an exceptional source of energy that are capable of initiating chemical reactions without the need for catalysts, high temperature or high pressure. The high kinetic energy and penetrating nature of the electrons provide significant benefits over typical chemical methods.

This technology is currently used in an array of industries and common consumer products. Accelerator sales eclipse \$2B annually providing an estimated added value to products worth more than $\$ 500 \mathrm{~B}$ every year.

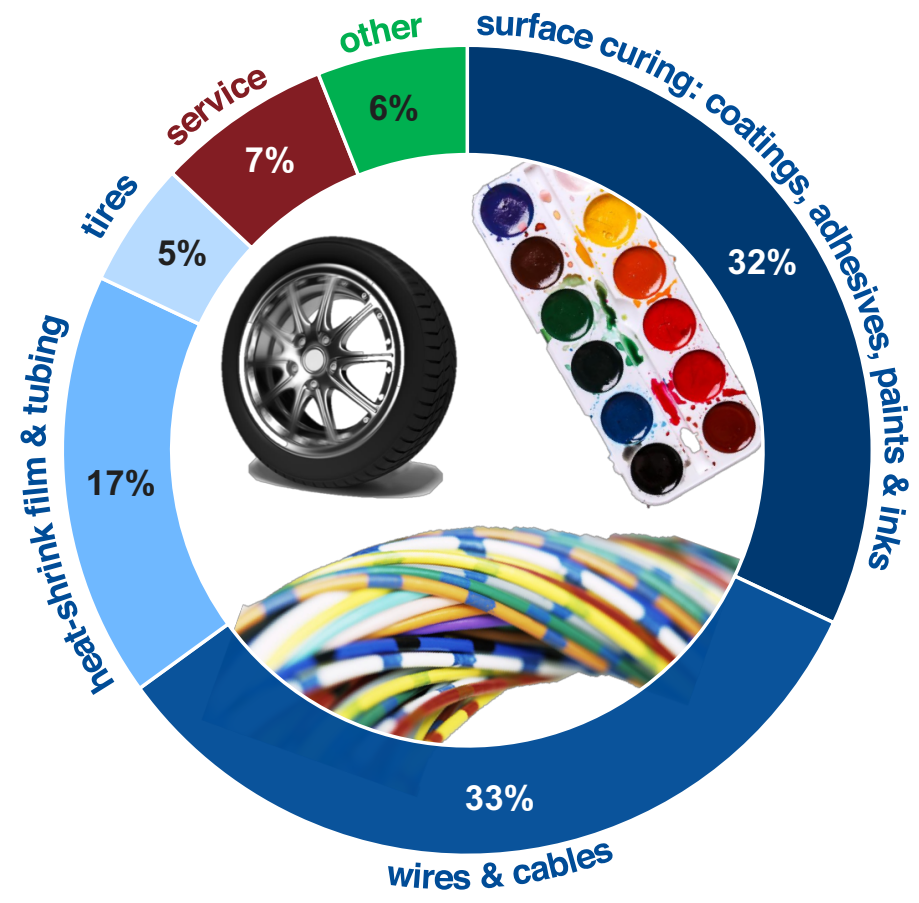

Current End-use Market Distribution of Electron Beam Industrial Applications

$87 \%$ of e-beam process involves crosslinking, represented by the applications in blue segments

Image source: IAEA Working Material on Industrial Electron Beam Processing

\section{Current E-beam Applications} Advantages

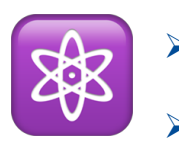

Improve physical \& chemical properties of existing materials

Enable new material synthesis routes

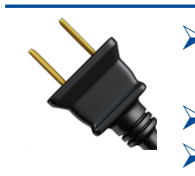

Eliminate high temperature \& high pressure

Continuous operation

$>$ Increase process yield

Estimated savings compared to conventional
technology
$>\begin{aligned} & \text { Tire industry }-16 \% \text { of manufacturing cost per } \\ & \text { tire [1] }\end{aligned}$
$>\begin{aligned} & \text { Cable insulation }-18 \% \text { of avg. cost per } \mathbf{k m} \text { of } \\ & \text { product }[2]\end{aligned}$
Curing $-25-65 \%$ of manufacturing cost [3]

[1] S. Tagawa et al., "Economic Scale of Utilization of Radiation (I): Industry. Comparison between Japan and the U.S.A.," Journal of Nuclear Science and Technology, vol. 39, no. 9, pp. 1002-1007, 2002.

[2] J. H. Bly, "Electron beam processing of power cable insulation," Radiation Physics and Chemistry (1977), vol. 18, no. 5, pp. 1331-1340, 1981.

[3] "Electron Beam Curing," Energy Program. [Online]. Available: http://e3tnw.org/ltemDetail.aspx?id=466.

\section{Drawbacks to current e-beam accelerators}

\section{Significant power consumption}

A large part of the input power in current accelerators is lost because of inefficient and outdated accelerator technologies.

\section{Slow throughput rates}

To avoid overheating, conventional accelerators operate in a "pulsed" mode with the beam ON only $2 \%$ of the time.

\section{Large footprint}

Requirements for cooling and shielding include large size components that limit portability.

Cost

With conventional accelerator technology, operating costs are high because of large use of power. 


\title{
Future of industrial chemistry
}

\author{
Illinois Accelerator Research Center
}

\section{Investigate}

Since the field of high-energy physics demands accelerators with ever increasing capabilities, significant effort has been put into developing, high-energy, high-power, high-efficiency and highly reliable accelerators at Fermi National Accelerator Laboratory.

Fermilab brings over 50 years of accelerator research, innovation, and application expertise to driving new industry solutions.

The lab is a go-to partner to federal agencies and members of industry for understanding and evaluating market opportunities in accelerator technology.

IARC is conducting studies to assess the current status of electron beam processing technology and to identify novel industrial applications where resulting properties are unique or enhanced relative to conventional processing.

\section{Innovate}

Leveraging Fermilab's intellectual property and other technology assets, the lab pushes the boundaries and advantages of electron accelerator use in specific industries.

We have developed new accelerator technologies which hold a number of advantages over conventional technologies. This innovation will provide solutions towards commercial needs that have either has little success or are excessively costly.

The novel design coupled with the portable nature of the accelerator will allow for increased application in industrial chemistry.

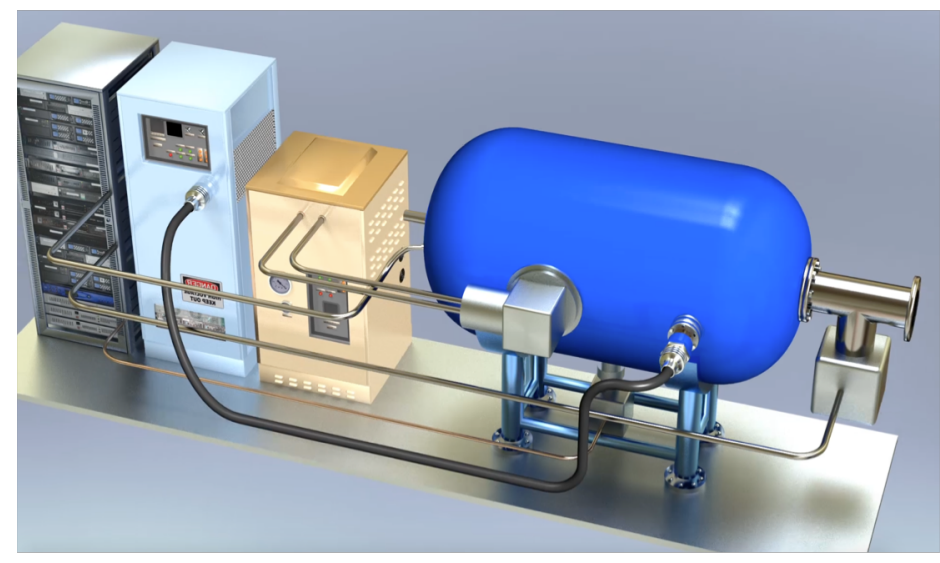

Novel compact electron beam accelerator design

\section{Demonstrate}

Proof-of-concept studies for research and validation of new applications can be conducted using IARC's demonstration accelerator.

Current projects include hardening pavement and improving wastewater treatment.

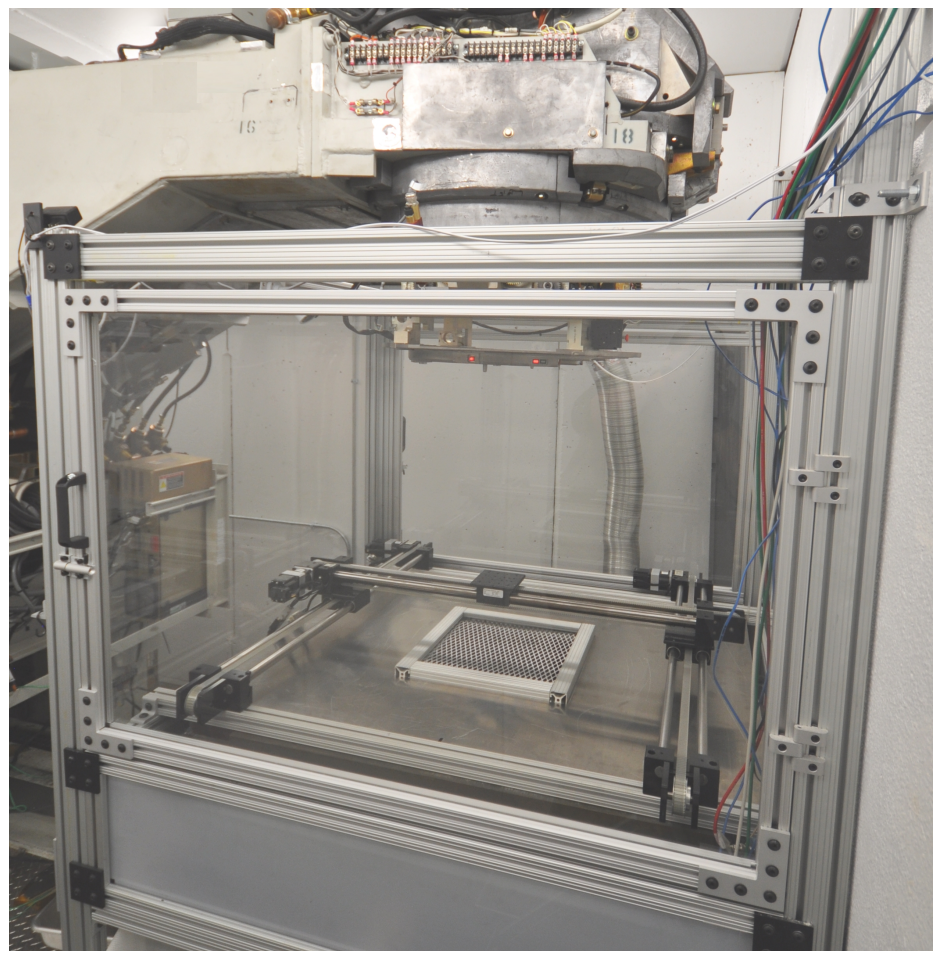

The Accelerator Applications Development and Demonstration (A2D2) is a tool for verifying electron beam proof-of-concept for individual applications

\section{Emerging e-beam applications}

- Biofuel production - scission of cellulose

- Fluorochemistry - radiation grafting on fluoropolymers

- Petroleum industry - chain scission of hydrocarbons

- Carbon fiber modification - adhesion by crosslinking

- Composites curing - crosslinking

- Stack gas treatment - radiolysis of gases

- Water treatment - decomposition of organics 